\title{
Interesting facts about PCOS: why does PCOS require 30 days of recognition?
}

Keywords: polycystic ovary syndrome, infertility, genetic factors, overweight

\section{Commentary}

September is Polycystic Ovary Syndrome Awareness Month, 30day recognition of the symptoms, treatment and hope for those with this syndrome.

Polycystic ovary syndrome (PCOS) is a hormonal, reproductive disorder that affects one in 10women of childbearing age. World Health Organization estimates 116million women worldwide to be affected by PCOS. The exact cause is multifactorial with multiple environmental and genetic factors fostering its development.

The classic signs associated with PCOS include weight gain, acne, irregular or non-existent periods, excessive hair growth in unwanted places and infertility.

\section{Here are the interesting facts}

Many women with PCOS are obese or overweight. Gaining weight in the abdominal area specifically is more common in women with PCOS. Specifically, if your abdominal circumference is more than $88 \mathrm{~cm}$ (35inches), then it could be a subtle sign you have may have PCOS. In addition, women who have PCOS sometimes develop a dark ring around their neck and creases of the skin, aka acanthosis nigricans. This happens due to insulin resistance. Interestingly there may be a male version of PCOS. Hormonal and metabolic abnormalities are described in male family members of women with PCOS. These men tend to be bald before the age of 35 . They may also be at a higher risk of type II diabetes, heart disease and benign prostate hyperplasia later in life. And finally, though many women with PCOS have irregular periods, their periods may get regular with reproductive aging.

Not every woman with PCOS has unwanted hair. Not everyone has irregular periods. In fact, some women with PCOS ovulate regularly. Not every woman with PCOS is overweight.

And there is no one single test for PCOS, which is why it is important to see a reproductive endocrinologist, aka a fertility specialist, to rule out other causes that can present with similar symptoms and confirm your diagnosis.

PCOS is diagnosed based on your gynecological history, an ultrasound to count the baseline follicles on your ovaries as well as a hormonal checkup which includes tests to check whether there is an underlying thyroid or pituitary problem or another testosteronerelated issue.

\section{While there is no cure for PCOS, it can be treated}

Weight loss with a healthy lifestyle that includes a balanced diet and regular exercise can have a positive effect on the endocrine system, particularly in cases when a woman is overweight or obese. Birth control pills are often prescribed to correct the hormone
Volume 5 Issue 6 - 2017

\author{
Sinem Karipcin \\ Reproductive Endocrinology and Infertility Clinical Fellow at \\ Weill Cornell Medical College, USA \\ Correspondence: Sinem Karipcin, Reproductive \\ Endocrinology and Infertility Clinical Fellow at Weill Cornell \\ Medical College, USA, Email dr.sinemkaripcin@gmail.com
}

Received: September 25, 2017| Published: December I, 2017

imbalance and lower the testosterone, which generally improves acne and unwanted hair growth and regulates your period. Additionally, Metformin, a medication that helps regulate blood sugar, is sometimes prescribed in conjunction with birth control pills or on its own for women who are pre-diabetic or diabetic.

If you are planning a family, there is good news: women with PCOS tend to have a higher ovarian reserve, which means that you may have a longer reproductive life and reach menopause later than the usual time. Also, if you were to undergo IVF treatment, you may have a larger number of eggs harvested.

The risk of pregnancy complications is increased in women with PCOS. Women with PCOS have more miscarriages. The rate of gestational diabetes, preeclampsia, preterm birth is also higher in women with PCOS compared to general population. Most of the complications are related to the metabolic consequences of PCOS.

As a fertility specialist, I see many women worried about their fertility potential because of the PCOS diagnosis. Actually, most normal weight women who aren't ovulating regularly due to PCOS, a healthy pregnancy can be achieved easily and inexpensively with an oral ovulation induction medication.

PCOS can have a major effect on a woman's reproductive and general health. Even if you are not planning to have children, early diagnosis and treatment along with weight loss is important as it may reduce the risk of long-term complications such as type 2diabetes and heart disease. That in itself makes PCOS Awareness worthy of 30days of recognition.

\section{Acknowledgments}

None.

\section{Conflicts of interest}

The author declares that there are no conflicts of interest.

\section{Funding}

None. 